\title{
Expression and prognosis of the B7 family in acute myeloid leukemia
}

\author{
Wei Zhang ${ }^{1}$, Wenjing Zhang ${ }^{1}$, Lin Gui ${ }^{1}$, Xue Yan $^{1}$, Xuan Zhou ${ }^{1}$, Yongchao Ma ${ }^{1}$, Zhinan Yang ${ }^{1}$, Yu Fang ${ }^{1}$, \\ Hongmei Zhang ${ }^{2}$, Jinning Shi ${ }^{1}$ \\ ${ }^{1}$ Department of Hematology, The Affiliated Jiangning Hospital with Nanjing Medical University, Nanjing, China; ${ }^{2}$ Department of Blood \\ Transfusion, The Affiliated Jiangning Hospital with Nanjing Medical University, Nanjing, China \\ Contributions: (I) Conception and design: W Zhang, H Zhang, J Shi; (II) Administrative support: H Zhang, J Shi; (III) Provision of study materials or \\ patients: W Zhang, W Zhang, Y Ma; (IV) Collection and assembly of data: X Yan, X Zhou, Y Fang; (V) Data analysis and interpretation: W Zhang, \\ L Gui, Z Yang; (VI) Manuscript writing: All authors; (VII) Final approval of manuscript: All authors. \\ Correspondence to: Jinning Shi. Department of Hematology, The Affiliated Jiangning Hospital with Nanjing Medical University, Nanjing, China. \\ Email: s8j8n8@126.com; Hongmei Zhang. Department of Blood Transfusion, The Affiliated Jiangning Hospital with Nanjing Medical University, \\ Nanjing, China. Email: zhm.0619@126.com.
}

Background: B7 family molecules affect both immune responses and cancer progression via immunological and non-immunological pathways. However, the specific expression and prognostic value of B7 members in acute myeloid leukemia (AML) remains unclear; hence, an investigation using online bioinformatics databases is required.

Methods: In this study, we explored the expression of B7 molecules using the ONCOMINE, Gene Expression Profiling Interactive Analysis 2 (GEPIA2), and UALCAN databases, while the prognostic value of B7 molecules in AML was analyzed using the LinkedOmics, GEPIA2, UALCAN, and TCGAportal databases. Additionally, genetic alteration and gene co-expression analysis of the B7 family was performed via the cBioPortal and LinkedOmics databases, while functional and pathway enrichment analyses were conducted using the Metascape databases for Gene Ontology (GO) and Kyoto Encyclopedia of Genes and Genomes (KEGG) enrichment analyses.

Results: The message RNA (mRNA) levels of B7 family members varied in AML patients, and aberrant highly expressed B7 members were correlated with poor prognosis in AML, including B7.1, B7-DC, B7$H 3, B 7-H 5$, and $B 7-H 7$. B7-H6 acted as a protective molecule for overall survival (OS), while $B 7-H 1$ overexpression was inclined to predict poor prognosis. B7 family gene alteration occurred frequently in $\mathrm{AML}$, and the altered B7 group seemed to exhibit a trend towards worse OS. The co-expression genes and relative signaling pathways of the B7 family might be involved in oncogenesis and be associated with prognosis in AML.

Conclusions: Our study showed that aberrantly expressed B7 family molecules affected the prognosis of AML patients, and thus, could be promising prognostic biomarkers and new therapeutic targets.

Keywords: Co-stimulatory molecules; B7; acute myeloid leukemia (AML); prognosis; bioinformatics integration analysis

Submitted Aug 03, 2021. Accepted for publication Sep 09, 2021.

doi: $10.21037 / \mathrm{atm}-21-4255$

View this article at: https://dx.doi.org/10.21037/atm-21-4255 


\section{Introduction}

Acute myeloid leukemia (AML), a heterogeneous hematologic malignancy, is characterized by clonal expansion of abnormally differentiated blasts of myeloid lineage, which leads to the accumulation of immature progenitors in bone marrow, peripheral blood, and/or other tissues. This leads to impairment of normal haemopoiesis, resulting in severe infections, anemia, and haemorrhage (1). In recent years, the application of risk-adapted management strategies based on consensus risk stratification guidelines have incorporated a growing number of molecular aberrations, coupled with the development of a series of new drugs and normalization cancer immunotherapy, have improved AML patient outcomes (1-3). However, the therapeutic options for refractory/relapsed patients and unfit populations remained challenges for AML overall management, especially within the evolvement of genomical heterogeneity under treatment pressure (4). Therefore, the significance of identifying potential biomarkers for AML has been defined in the literature, which aids in recognizing patients with poor prognosis, distinguishing biological driver genes, as well as performing targeted therapies and new normalization cancer immunotherapies.

B7 family molecules have important functions in adaptive immune responses and oncogenesis, delivering co-stimulatory or co-inhibitory signals combined with their ligands, and also participating in cancer progression and chemoresistance via non-immunological pathways (5). Ten B7 family members have been identified, including B7.1 (CD80), B7.2 (CD86), B7-DC (CD273, PD-L2, PDCD1LG2), B7-H1 (CD274, PD-L1), B7-H2 (CD275, ICOSLG, ICOSL), B7-H3 (CD276), B7-H4 (VTCN1), B7-H5 (VSIR, VISTA, C10orf54), B7-H6 (NCR3LG1, DKFZp686O24166), and B7-H7 (HHLA2) (6).

Previous studies have demonstrated that various B7 molecules are aberrantly expressed in many solid tumors and AML, and are associated with immune disorders and poor prognosis $(6,7)$. One study reported that immunophenotyping in $34 \mathrm{AML}$ patients showed lowly expressed cluster of differentiation 80 (CD80) and variably expressed CD86 in most AML cells (8). Another study reported that the bone marrow of 107 AML patients exhibited an increased frequency of regulatory $\mathrm{T}$ cells, was more frequently positive for programmed cell death-ligand 1 (PD-L1) in the blasts of tumor protein p53 (TP53)mutated patients, and had higher levels of programmed cell death-1 (PD1)-positive/OX40 [TNF receptor superfamily member 4 (TNFRSF4)]-positive T cells (9). Another study investigated the peripheral blood mononuclear cells of myelodysplastic syndrome and AML patients, which were observed for upregulated $P D-L 1$ and $P D-L 2$ mRNA expression, with negatively correlated to the response to hypomethylating agents and survival (10). Inducible $\mathrm{T}$ cell costimulator ligand (ICOSL) expression by AML cells can promote the expansion of regulatory $\mathrm{T}$ cells and predict poor survival in AML patients (11). Moreover, B7-H3 expression in acute leukemia is higher in CD34+ cases and high-risk karyotype patients, and predicts an unfavorable outcome (12). High levels of VISTA [V-set immunoregulatory receptor (VSIR)] expression have also been observed in myeloid-derived suppressor cells of peripheral blood; this was found to be positively correlated with PD-1 expression in T cells and inhibit the T cell response in AML (13). B7-H6 and B7$\mathrm{H} 7$ was detected in human haematological tumor cells, and $T$ cell-induced cytotoxicity was found to increase when $\mathrm{T}$ cells armed with the bispecific antibody anti-CD3 and anti-B7-H6 target to B7-H6+ tumor cells (14). Our previous studies had found that the protein expression of B7 family negative costimulatory molecules, B7-H1, B7$\mathrm{H} 3$ and $\mathrm{B} 7-\mathrm{H} 4$, abnormally overexpressed in AML cell lines compared with the peripheral blood mononuclear cells of healthy volunteers, moreover the subcellular distribution was most in nucleus and cytoplasm (15), and the negative costimulatory molecule $\mathrm{B} 7-\mathrm{H} 3$ could promote AML cell line U937 cell progression in vitro and in vivo (16). Thus, B7 family molecules might be potential therapeutic targets in AML. Although there have been several studies on the expression and effect of B7 molecules in AML patients, the comprehensive understanding for expression characteristics and prognostic significance of distinct ten B7 family members in a whole AML cohort have not yet been clearly elucidated. Meanwhile, there were few researches explored the co-expression genes, upstream regulators and downstream effectors of B7 family in AML.

In this study, online bioinformatics databases were utilized to investigate the expression of various B7 family members, explore the prognosis value of $\mathrm{B} 7$ molecules in AML patients, perform genetic alteration and gene co-expression analyses, and examine the regulation of $\mathrm{B} 7$-related pathways. This will provide the basis for enriching prognostic scoring system and the clinical application of B7 molecules targeted therapies in AML. We present the following article in accordance with the REMARK reporting checklist (available at https://dx.doi. org/10.21037/atm-21-4255). 


\section{Methods}

\section{Ethical statement}

This study was performed in accordance with the Helsinki Declaration (as revised in 2013) and was approved by the Institutional Ethics Committee of The Affiliated Jiangning Hospital with Nanjing Medical University. Written informed consent had already been provided by the study subjects; thus, the patient characteristics data could be retrieved from the online public databases.

\section{ONCOMINE database}

ONCOMINE (https://www.oncomine.org) is an online cancer microarray database and data-mining platform that aims to promote genome-wide expression analyses and facilitate the discovery of novel molecules for the identification of medical conditions and targeted therapies (17). In this study, the ONCOMINE database was applied to analyze the transcriptional expression level of the B7 family in different cancers and relative normal controls, and the Student's $t$-test was utilized for differential expression (cut-off values: $\mathrm{P}=0.01$, fold change: 1.5 ).

\section{GEPIA2 database}

GEPIA2 (http://gepia2.cancer-pku.cn) database is an updated and enhanced version of GEPIA, and contributes to gene expression quantification analyses based on normal and tumor samples from 198,619 isoforms and 84 cancer subtypes from The Cancer Genome Atlas (TCGA) and GenotypeTissue Expression (GTEx) databases. It provides customized analysis, including differential expression analysis, patient survival analysis, dimensionality reduction analysis, similar gene detection, correlation analysis, profiling plotting, and also supports the upload of researchers' own RNAsequencing (RNA-seq) data for comparison with the TCGA and GTEx samples $(18,19)$. In this study, the GEPIA2 dataset was adopted to analyze the differential mRNA expression of B7 family members in both AML and normal samples, and to assess the prognostic significance of B7 molecules in AML. The expression analysis was performed using the Student's $t$-test, and survival analysis was conducted using the log-rank test, with a cut-off value of $\mathrm{P}=0.05$.

\section{UALCAN database}

UALCAN (http://ualcan.path.uab.edu), an interactive web-portal, uses TCGA level 3 RNA-seq and clinical data from 31 cancer types to estimate the relative expression of target genes across tumor and normal samples. It also aids in evaluating the effect of gene expression and clinicopathological characteristics on survival (20). We used the UALCAN database to analyze the mRNA expression levels of B7 family members in AML based on the FrenchAmerican-British (FAB) classification, and to estimate the prognostic significance of B7 expression in AML. Differential expression was performed using the Student's $t$ test, with a cut-off value of $\mathrm{P}=0.05$.

\section{LinkedOmics database}

LinkedOmics (http://www.linkedomics.org) is the first multi-omics database, and integrates proteomics data generated by the Clinical Proteomic Tumor Analysis Consortium (CPTAC) on -32 cancer types from TCGA tumor samples. This database is a unique platform for discovering and comparing cancer multi-omics attribute associations (21). In this study, the LinkedOmics database was used to analyze the prognostic value of $\mathrm{B} 7$ gene expression and their negatively- and positively-correlated significant genes in AML. Survival analysis was performed using the log-rank test, and genes that were correlated with the B7 family were analyzed using Pearson's correlation test. The cut-off $\mathrm{P}$ value was 0.05 .

\section{TCGAportal database}

TCGAportal (http://www.tcgaportal.org), an open-access portal website, was designed by Shutan $\mathrm{Xu}$ from the Shaying Zhao Lab at the University of Georgia. Based on the samples in TCGA and GTEx databases, the TCGAportal displays the gene expression, performs differential function enrichment analysis, and predicts the bind form between a small molecule (such as drug molecule) and a receptor protein. Survival analysis of $\mathrm{B} 7$ family members in AML was performed using the log-rank test, with a cut-off value of $\mathrm{P}=0.05$.

\section{cBioPortal database}

cBioPortal (https://www.cbioportal.org) is a website used to interactively explore genetic alterations across samples, genes, and pathways, as well as subsequent analysis of these factors in terms of clinical outcomes (22). In this study, the cBioPortal database was used to analyze the genetic alteration, prognostic value, and relative gene alteration 
frequency of B7 family members in the altered and unaltered B7 AML groups. The mRNA expression z-scores threshold was defined as \pm 1.8 . The log-rank test was employed for survival analysis, with a cut-off value of $\mathrm{P}<0.05$.

\section{Metascape database}

As a web-based integrated portal, Metascape (http:// metascape.org) combines the functions of membership search, gene annotation, interactome analysis, and functional enrichment, involving over 40 independent knowledge bases, including GO and KEGG analyses (23). With 20 selected similar expression genes significantly associated with each B7 family molecule detected using the GEPIA2 dataset, GO and KEGG enrichment analyses with B7 molecules and their relative genes were then performed in the Metascape database.

\section{Statistical analysis}

The Student's $t$-test was utilized for differential expression of B7 family in different cancers and relative normal controls. Survival analysis was conducted using the log-rank test. The genes correlated with the B7 family were analyzed using Pearson's correlation test. A value of $\mathrm{P}<0.05$ was considered statistically significant. The statistical analysis was performed by above online bioinformatics databases.

\section{Results}

\section{The transcriptional level of $B 7$ family members in $A M L$} patients

Using the ONCOMINE database, the comparison between the transcriptional level of different B7 molecules in 20 types of cancers and the corresponding normal controls was analyzed (Figure 1 and Table 1). Specifically, leukemia patients were found to be associated with downregulated mRNA expression of B7.1 and B7-H5 and upregulated/ downregulated mRNA expression of $B 7.2$ and $B$ 7- $H 2$ (increasing in some datasets, while decreasing in the others). Additionally, no marked differences were identified between leukemia patients and normal samples in the mRNA levels of B7-DC, B7-H1, B7-H3, B7-H4, B7-H6, and B7-H7. Compared with the normal controls, $B 7.2$ has been shown to be over-expressed in B-cell childhood acute lymphoblastic leukemia (ALL) (fold change: 3.393, $\mathrm{P}=0.009$ ) (24), T-cell prolymphocytic leukemia (fold change: $2.825, \mathrm{P}=0.004$ ), and chronic adult T-cell leukemia/lymphoma (fold change: 2.290, $\mathrm{P}=0.002)(25,26)$. In the Durig Leukemia dataset, the mRNA expression of $\mathrm{B} 7-\mathrm{H} 2$ has been shown to exhibit a higher expression in T-cell prolymphocytic leukemia (fold change: $3.678, \mathrm{P}=0.008)(25)$. Meanwhile, $B 7-H 2$ has been shown to be overexpressed in chronic lymphocytic leukemia (fold change: $3.579, \mathrm{P}=1.07 \mathrm{E}-88$ ), B-cell childhood ALL (fold change: $3.109, \mathrm{P}=1.32 \mathrm{E}-74$ ), pro-B ALL (fold change: 2.865, $\mathrm{P}=1.07 \mathrm{E}-22$ ), and $\mathrm{B}$-cell ALL (fold change: 2.607, $\mathrm{P}=1.13 \mathrm{E}-34)$ (27). Also, in Andersson Leukemia dataset, $-B 7-H 2$ was found to be over-expressed in B-cell ALL patients (fold change: $2.739, \mathrm{P}=3.67 \mathrm{E}-15$ ) (28).

Next, the mRNA expressions of B7 family members between AML and normal samples were compared using the GEPIA2 database (Figure 2). Specifically, compared with the normal controls, AML samples exhibited marked increases in $B 7.2$ and $B 7-H 5$ expression, and significant reductions in $B 7.1, B 7-H 3$, and $B 7-H 6$ expression $(\mathrm{P}<0.05)$. Meanwhile, there were no notable differences in the mRNA expression of B7-DC, B7-H1, B7-H2, B7-H4, and B7-H7.

Also, we used the UALCAN database to further explore the different mRNA expressions of $\mathrm{B} 7$ molecules in various FAB subtypes of AML patients (Figure 3). The mRNA expression of $B 7.1$ and $B 7-H 4$ was not significantly differences among the FAB subtypes (M0-M7). B7.2 mRNA expression was markedly elevated in M4 and M5, and was lower in M3 and M7. B7-DC transcriptional expression was higher in M6 and lower in M3. The mRNA level of $B 7-H 1$ was lower in M3 and M5. The transcriptional level of $B 7$ $H 2$ was decreased in M3, M4, and M5, and was upregulated in the other AML subtypes. B7-H3 mRNA expression was elevated in $\mathrm{M} 3$ and $\mathrm{M} 5$, and reduced in M0, M1, M2, and M4. B7-H5 mRNA expression was ascending in M4, M5, and M6, and lower in M3. The transcriptional expression of B7-H6 was higher in M3. The mRNA level of $B 7-H 7$ was lower in M3, M5, and M7, and higher in M4.

\section{The prognostic significance of $m R N A$ expression of $B 7$ molecules in AML patients}

The prognostic value of $\mathrm{B} 7$ molecules in AML was analyzed using the LinkedOmics, GEPIA2, UALCAN, and TCGAportal databases, respectively (Figure 4). The prognostic value of different $\mathrm{B} 7$ members in $\mathrm{AML}$ varied, and the prognostic significance of a specific B7 molecule was not identical among the different databases. The LinkedOmics database analysis showed that the decreased $B 7-H 6$ and increased $B 7-H 5$ and $B 7-H 7$ expressions in 

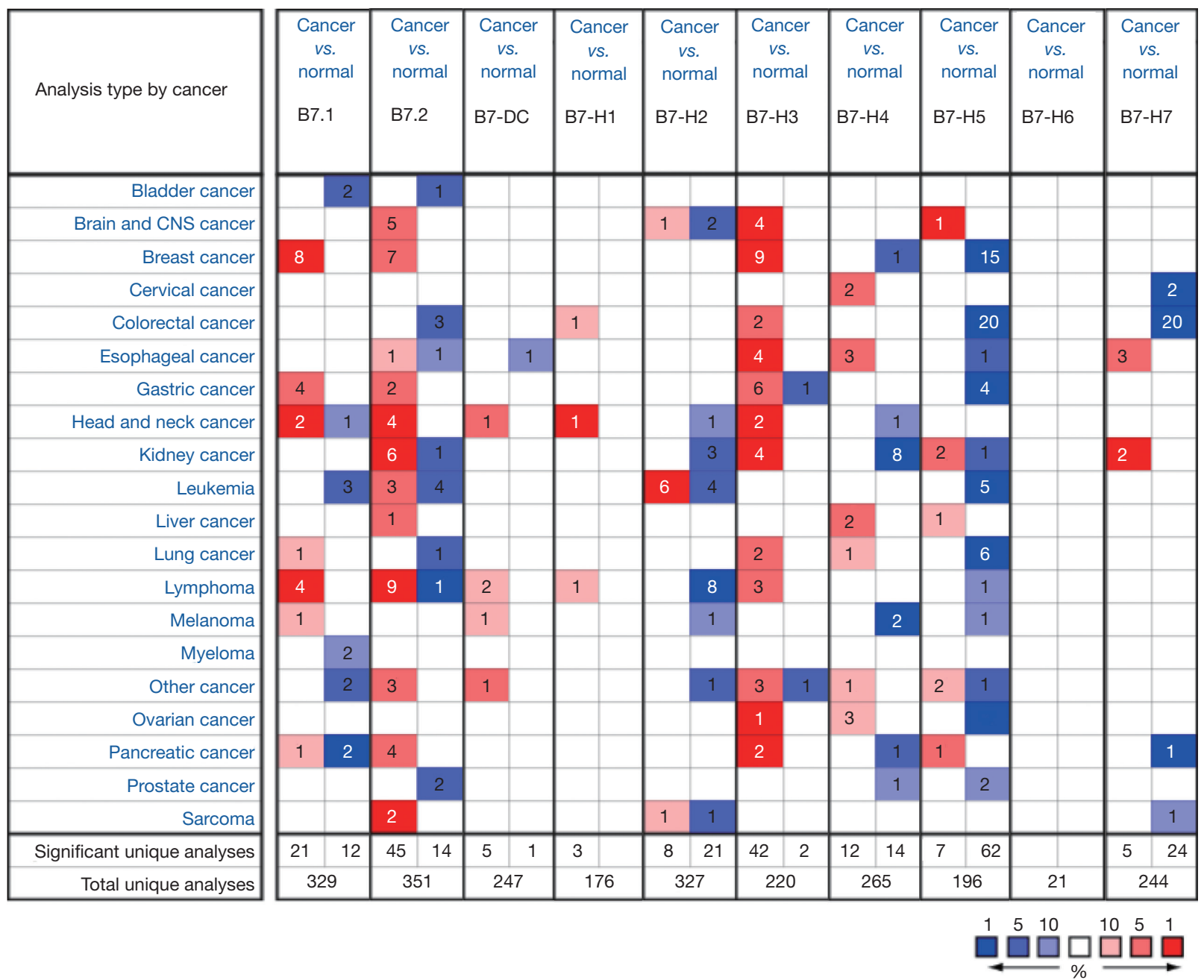

Figure 1 The transcriptional expression of B7 molecules in 20 cancer types (ONCOMINE).

Table 1 Significant changes in the transcriptional level of B7 family members between leukemia and normal controls (ONCOMINE)

\begin{tabular}{|c|c|c|c|c|c|}
\hline B7 members & Types of leukemia (vs. normal) & Fold Change & $P$ value & $t$ test & Reference \\
\hline \multirow{2}{*}{ B7.2 } & T-cell prolymphocytic leukemia & 2.825 & 0.004 & 3.162 & Dürig Leukemia (25) \\
\hline & Chronic adult T-cell leukemia/lymphoma & 2.290 & 0.002 & 3.188 & Choi Leukemia (26) \\
\hline \multirow[t]{5}{*}{$\mathrm{B} 7-\mathrm{H} 2$} & T-cell prolymphocytic leukemia & 3.678 & 0.008 & 2.951 & Dürig Leukemia (25) \\
\hline & B-cell childhood acute lymphoblastic leukemia & 3.109 & $1.32 \mathrm{E}-74$ & 23.573 & Haferlach Leukemia (27) \\
\hline & Pro-B acute lymphoblastic leukemia & 2.865 & $1.07 \mathrm{E}-22$ & 13.089 & Haferlach Leukemia (27) \\
\hline & B-cell acute lymphoblastic leukemia & 2.607 & $1.13 \mathrm{E}-34$ & 14.907 & Haferlach Leukemia (27) \\
\hline & B-cell acute lymphoblastic leukemia & 2.739 & $3.67 \mathrm{E}-15$ & 14.202 & Andersson Leukemia (28) \\
\hline
\end{tabular}




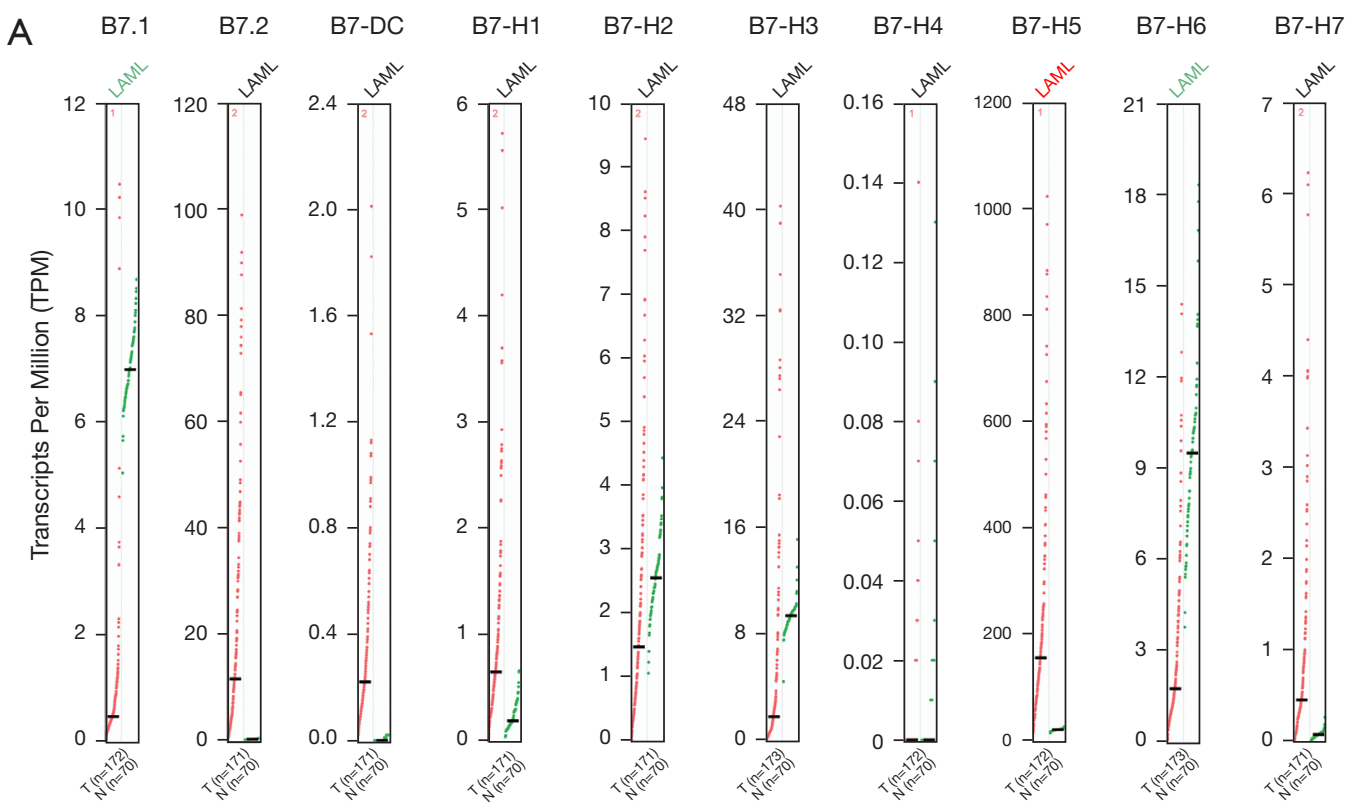

B

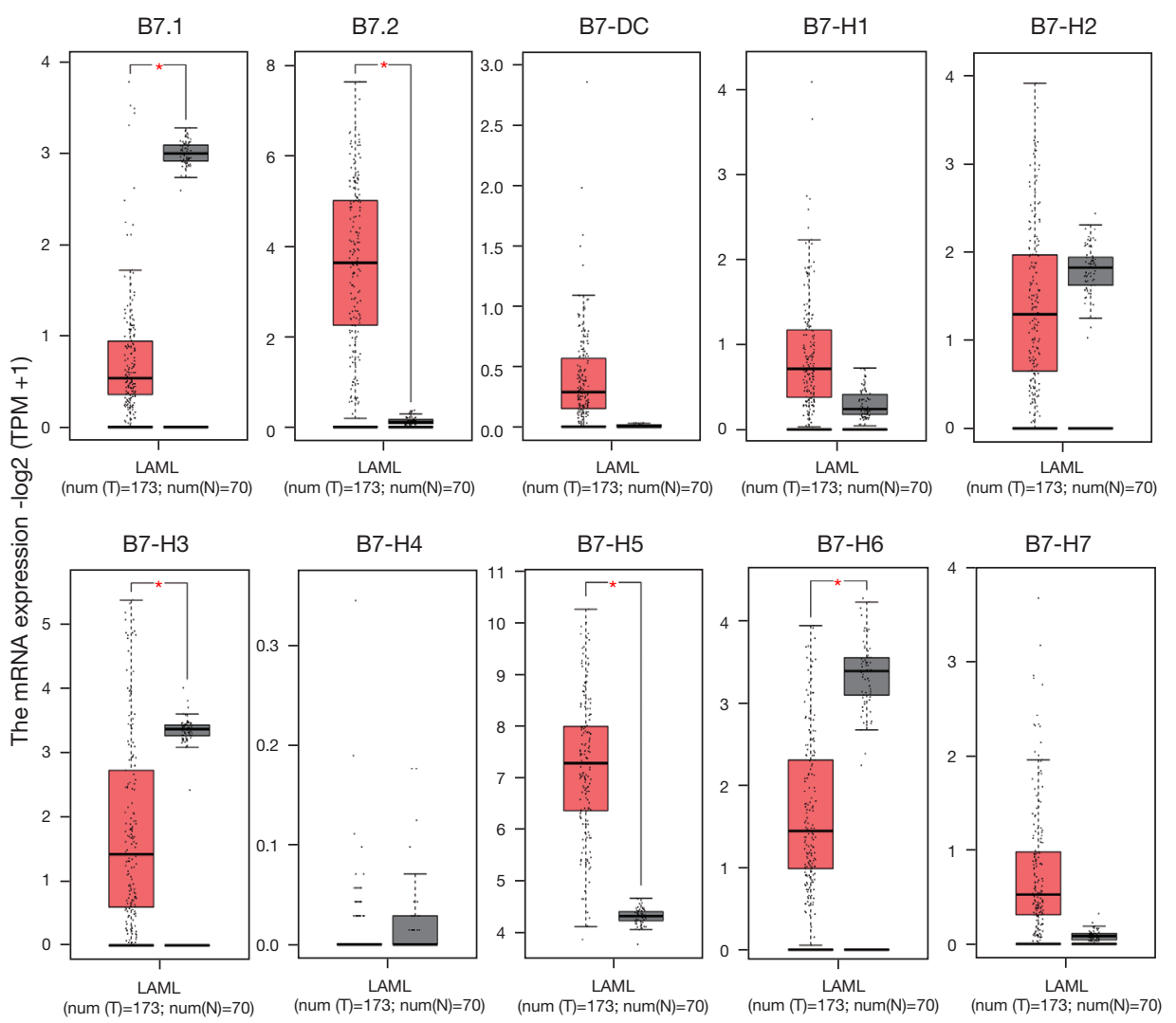

Figure 2 The mRNA expression of B7 molecules in AML and normal samples (GEPIA2). (A,B) The transcriptional level of B7 family members in AML compared with normal samples. LAML: AML; T: tumor (the AML samples); N: normal (the normal controls). *, it represents that the $\mathrm{B} 7$ family expression is statistically significant compared to the normal controls, $\mathrm{P}<0.05$. AML, acute myeloid leukemia. 

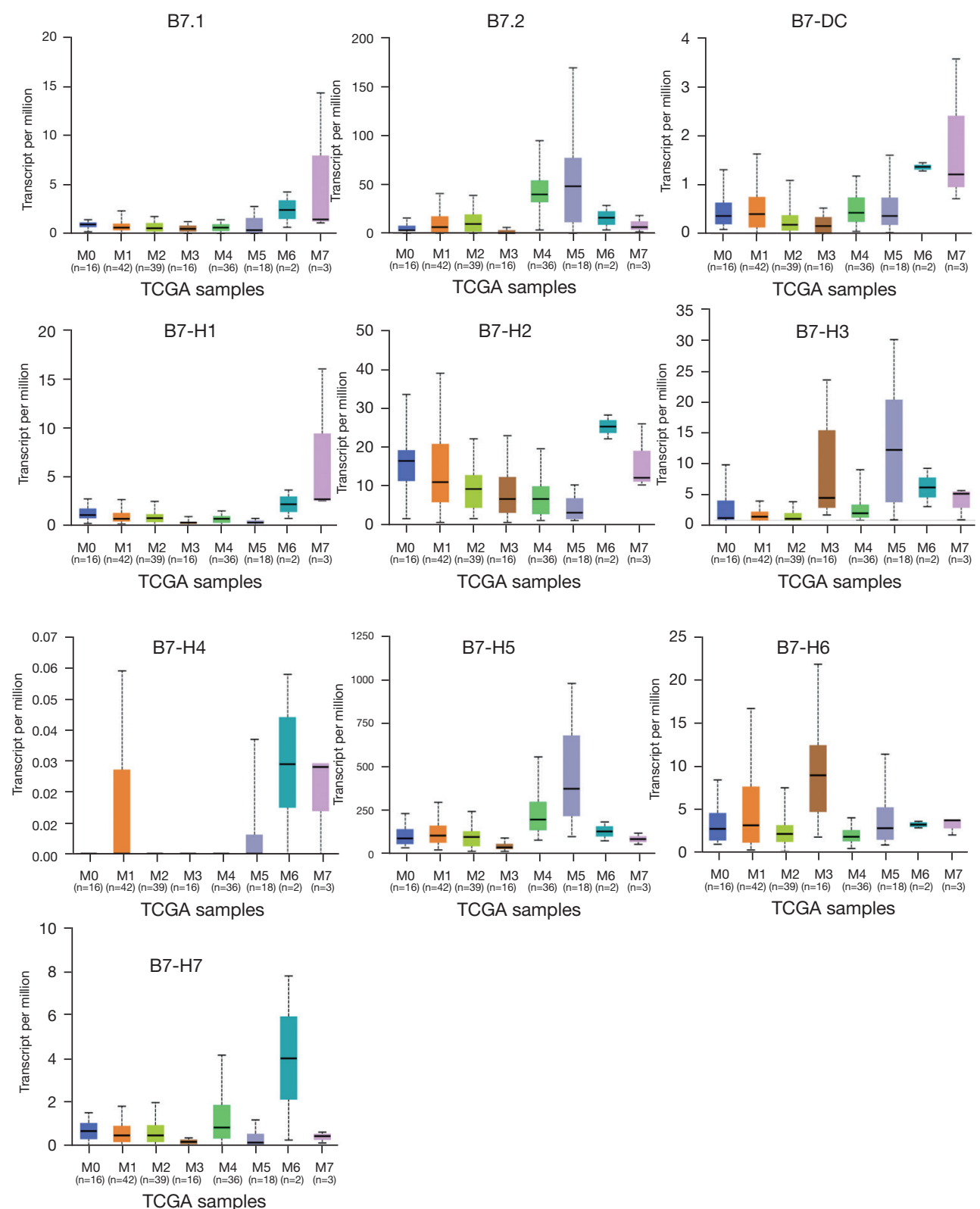

Figure 3 The mRNA expression levels of B7 molecules in AML based on FAB classification (UALCAN). TGCA, The Cancer Genome Atlas; AML, acute myeloid leukemia; FAB, French American-British.

AML were associated with poor overall survival (OS), while patients who had a high expression of $B 7.1$ and $B 7$ $H 1$ tended to have a poor prognosis, although this was not statistically significant $(\mathrm{P}>0.05)$. The GEPIA2 database analysis showed that poor OS was related to increased $B 7$ $H 3$ expression, and was more likely to occur in high $B 7$ $H 1, B 7-H 5$ and $B 7-H 7$ expression groups, however no significant statistical differences were observed $(\mathrm{P}>0.05)$. In the UALCAN database, a worse prognosis was identified in
AML patients with high expressions of $B 7.1, B 7-D C$, and $B 7-H 5$, and was more likely in the $B 7-H 3$ overexpression group, although this was not statistically significant $(\mathrm{P}>0.05)$. In TCGAportal database, a poor prognosis of AML was found to be related to high mRNA expression levels of $B 7-D C, B 7-H 3$ and $B 7-H 5$; and it tended to be in the upregulated $B 7-H 1$ and $B 7-H 7$ groups and in the reduced $B 7-H 6$ group, although the respective differences were not statistically significant $(\mathrm{P}>0.05)$. 

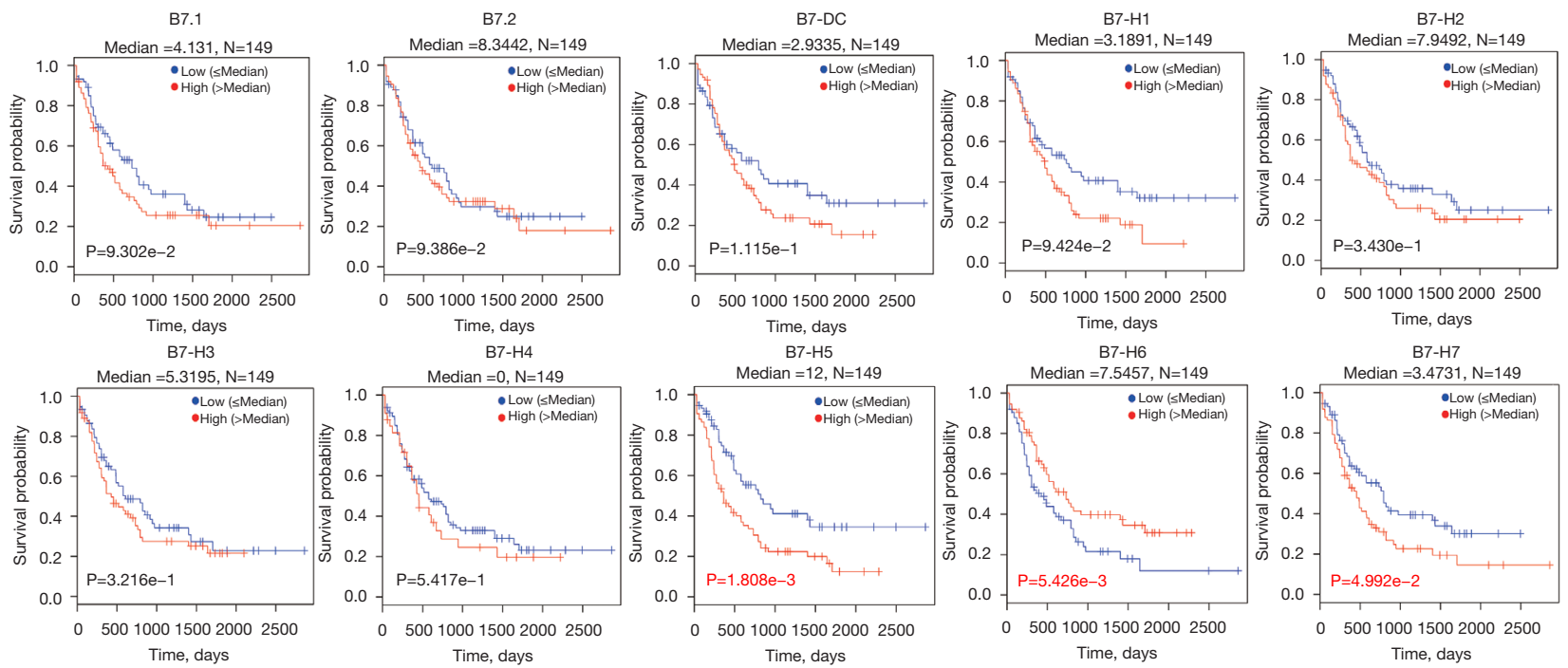

B7.1

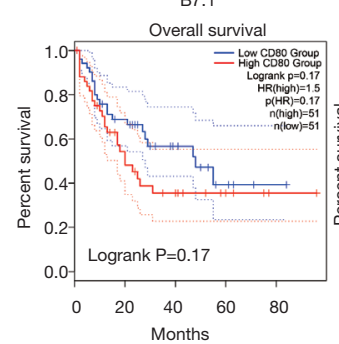

B7.2
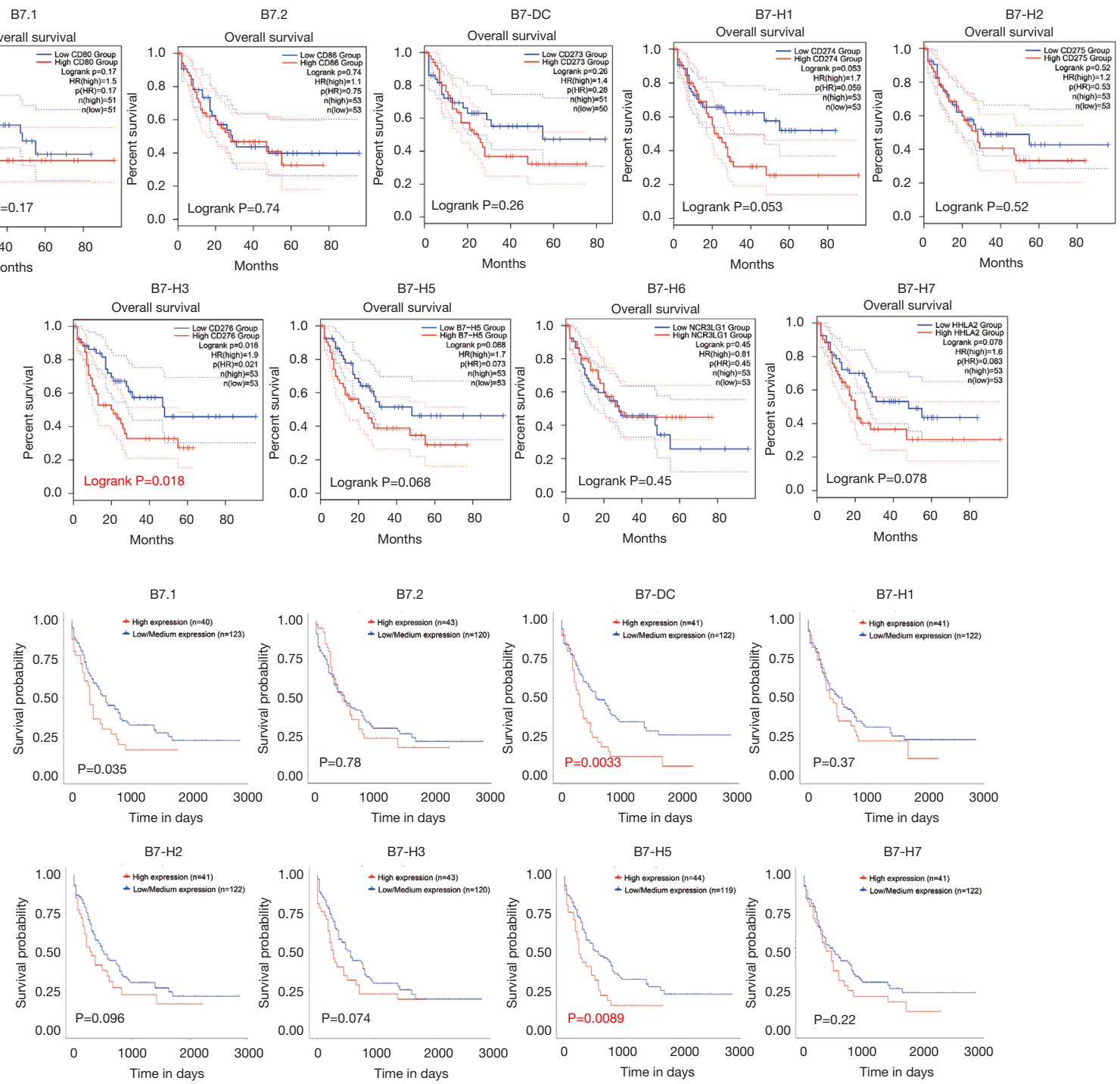

(c) Annals of Translational Medicine. All rights reserved. 

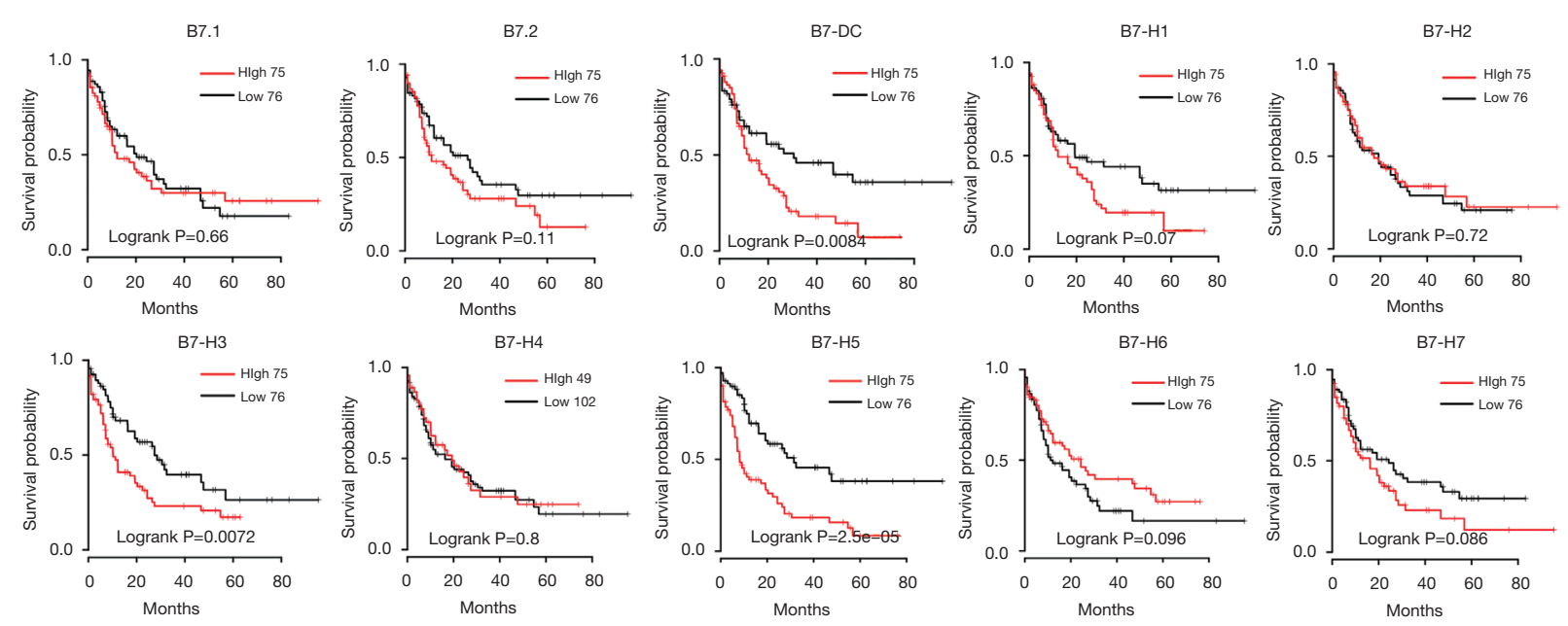

Figure 4 The prognostic value of B7 molecules expression in AML samples. Prognostic significance was analyzed using the LinkedOmics, GEPIA2, UALCAN, and TCGAportal databases, respectively. AML, acute myeloid leukemia; GEPIA2, Gene Expression Profiling Interactive Analysis 2.

\section{The genetic alteration of $B 7$ molecules in AML patients}

The genetic alteration of $\mathrm{B} 7$ molecules and their association with OS and other gene alterations in AML was analyzed using the cBioPortal database (Figure 5). The B7 family genes were altered in $53.76 \%$ of 173 AML cases, with $1.16 \%$ (2/173) amplification, 20.23\% (35/173) high mRNA expression, $31.21 \%$ (54/173) low mRNA expression, and $1.16 \%(2 / 173)$ multiple alteration, respectively. In $90 \mathrm{AML}$ patients with altered $\mathrm{B} 7$, there were 62 deceased cases, and the median OS was 12.03 months. Meanwhile, in the 71 unaltered B7 patients, 41 cases were deceased, and the median OS was 18.97 months. The OS differences between the altered and unaltered B7 groups were not statistically significant $(\mathrm{P}=0.0929)$. In addition, the relative gene copynumber amplification frequency in altered and unaltered $\mathrm{B} 7$ groups was different. Amplification of the KMT2A gene was often found in the unaltered $\mathrm{B} 7$ group, while other relative gene amplifications, such as PRDM15, ADARB1, AIRE, etc., were always identified in the altered $\mathrm{B} 7$ group. The most common gene mutations in the altered and unaltered B7 groups were NPM1 and FLT3, respectively.

\section{The significant co-expression genes correlated with B7 family members in AML patients}

The LinkedOmics database was used to analyze the correlated co-expression genes with B7 molecules in 173 AML patients (Figure 6). Heat maps demonstrated the top
50 genes that were positively and negatively correlated with B7 molecules, respectively (Figure 6A,6B). The correlation between the most positively or negatively correlated genes and the relative $\mathrm{B} 7$ molecules were displayed using scatter plots, respectively (Figure 6C,6D). The strongest positive co-expression genes with the $\mathrm{B} 7$ family were $G L I 2$, MPEG1, ҰAK2, BMP2K, SDK2, KIF1C, KCNG3, ITGB2, $X P O T$, and TIFAB, which correlated with B7.1, B7.2, B7$D C, B 7-H 1, B 7-H 2, B 7-H 3, B 7-H 4, B 7-H 5, B 7-H 6$, and B7$H 7$, respectively. Also, the strongest negative co-expression genes with these $\mathrm{B} 7$ molecules were UBE2I, KDM5B, QPCTL, VAMP8, AK2, B4GALT6, CMTM7, BCKDHB, $I T M 2 B$, and SPATA6, respectively.

\section{Functional and patbway enrichment analyses of B7 molecules and their associated genes in AML patients}

Subsequently, with the selection of 20 similar expression genes for a specific B7 molecule in AML through the GEPIA2 dataset, the Metascape database was applied for GO and KEGG enrichment analyses of B7 family members and their relative genes (Figure 7). The results showed that B7 molecules could affect the following processes in AML: oxidoreductase activity (GO:0016491), small hydrolase enzyme of guanosine triphosphate (GTPase)-mediated signal transduction (GO:0007264), positive regulation of the response to an external stimulus (GO:0032103), cellular response to an external stimulus (GO:0071496), lytic 


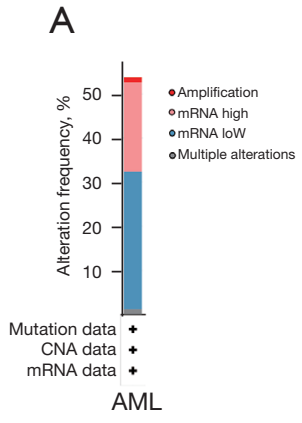

AML
B
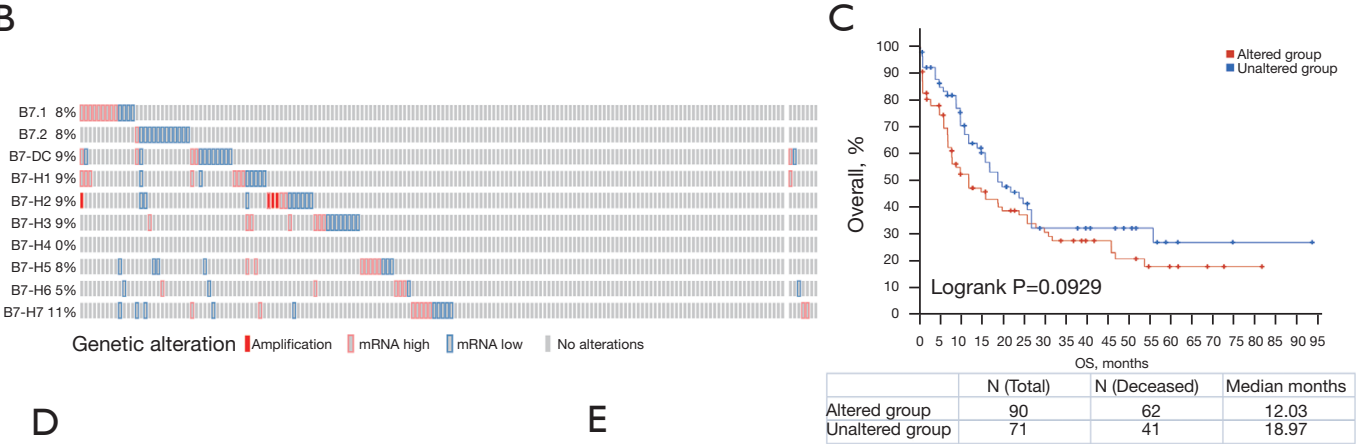

E
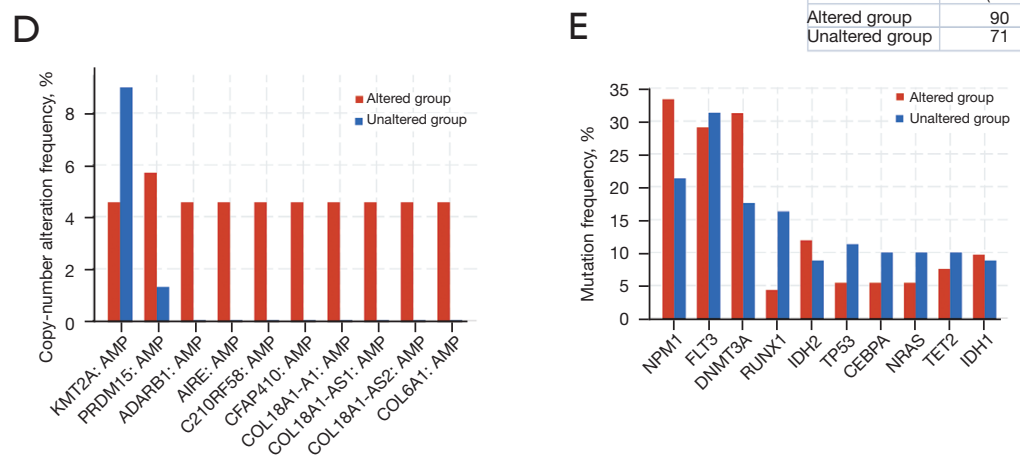

Figure 5 The genetic alteration of B7 molecules and their association with OS and other gene alterations in AML (cBioPortal). (A,B) Genetic alteration of the B7 family in AML; (C) the prognostic value of B7 genetic alteration in AML; (D,E) the relative gene alteration frequency in the altered and unaltered B7 groups in AML. AMP, copy-number amplification; OS, overall survival; AML, acute myeloid leukemia.

vacuole (GO:0000323), fatty acid transport (GO:0015908), cell redox homeostasis (GO:0045454), regulation of haemopoiesis (GO:1903706), regulation of the leukocyte apoptotic process (GO:2000106), cell activation involved in the immune response (GO:0002263), regulation of the immune effector process (GO:0002697), immune response-regulating signaling pathway (GO:0002764), the carbohydrate derivative biosynthetic process (GO:1901137), regulation of alpha-beta $\mathrm{T}$ cell activation (GO:0046634), response to virus (GO:0009615), regulation of the innate immune response (GO:0045088), cellular response to interferon-gamma (GO:0071346), phagocytic vesicle (GO:0045335), positive regulation of cytokine production (GO:0001819), and osteoclast differentiation (ko04380). The most frequently altered target transcription factors of the B7 family were ZNF528, ZSCAN23, MEF2D, YBX3, and $Z N F 669$, and the most frequent regulating genes towards B7 molecules were TRERF1, RELA, NFKB1, IRF1, and SPI1.

\section{Discussion}

The ligands and receptors of B7 family members affect
$\mathrm{T}$ cell immune regulation, and participate in various pathological states, such as cancers, transplantation, infection, and autoimmune diseases. There are three groups of B7 molecules divided phylogenetically: B7.1/B7.2/ CD28/CTLA4 and B7-H2/ICOS into group I; B7-H1/B7DC/PD-1 into group II; and B7-H3, B7-H4, and B7-H5/ CD28H into group III (29). Recent cancer immunotherapy strategies have undergone a paradigm shift from "immune enhancement" to "immune normalization" (3). A few studies reported on the expression state and prognostic role of B7 members in AML, such as B7-H1, B7-DC, B7$\mathrm{H} 2$, and B7-H3 (10-12). However, the expression patterns, prognostic value, genetic alteration, and other related genes and pathways of $\mathrm{B} 7$ molecules in AML patients have not been comprehensively elucidated. Thus, we performed a bioinformatics analysis in order to enhance the accuracy of prognosis evaluation and guide better designs for normalized immunotherapies in AML.

Previous studies have shown the following: (I) CD80 is lowly expressed and CD86 is variably expressed in most AML cells (8); (II) upregulated $P D-L 1$ and $P D-L 2$ mRNA expression was present in the peripheral blood mononuclear cells of AML cases (10); (III) the expression of ICOSL, B7- 
A
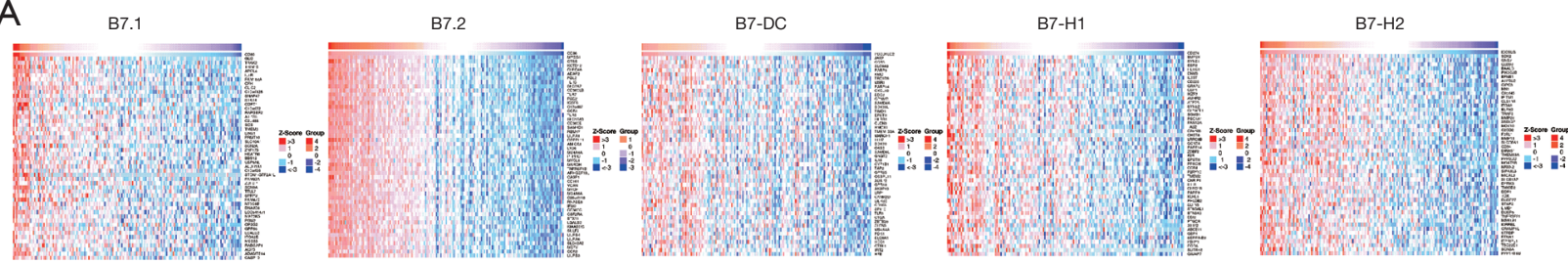

$\mathrm{B} 7-\mathrm{H} 3$
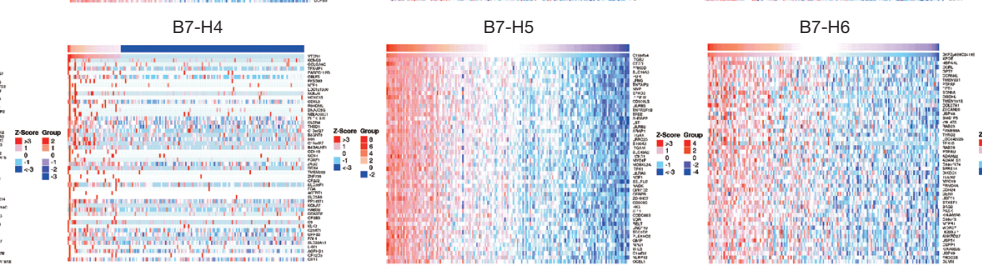

$\mathrm{B} 7-\mathrm{H} 7$

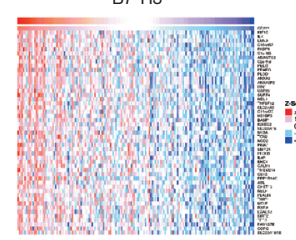

RAd

B

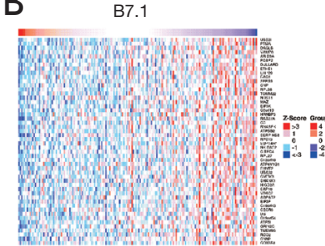

B7.2
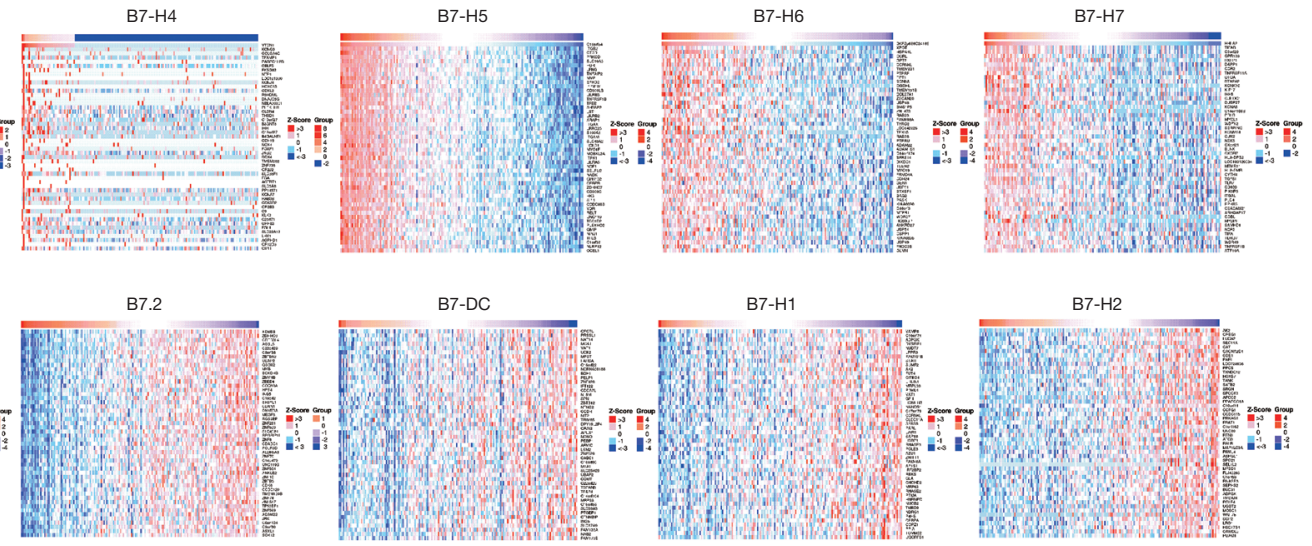

$\mathrm{B} 7-\mathrm{H} 3$

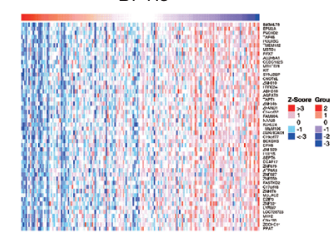

C Pearson-correlation: 0.592

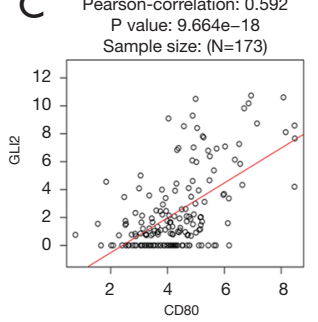

Pearson-correlation: 0.6257 $P$ value: $9.505 \mathrm{e}-20$

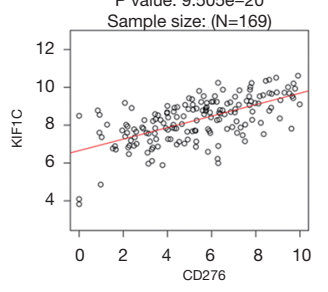

$\mathrm{B} 7-\mathrm{H} 4$

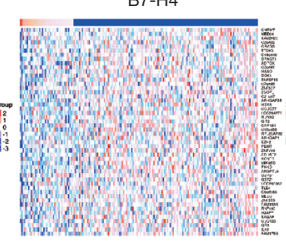

B7-H5
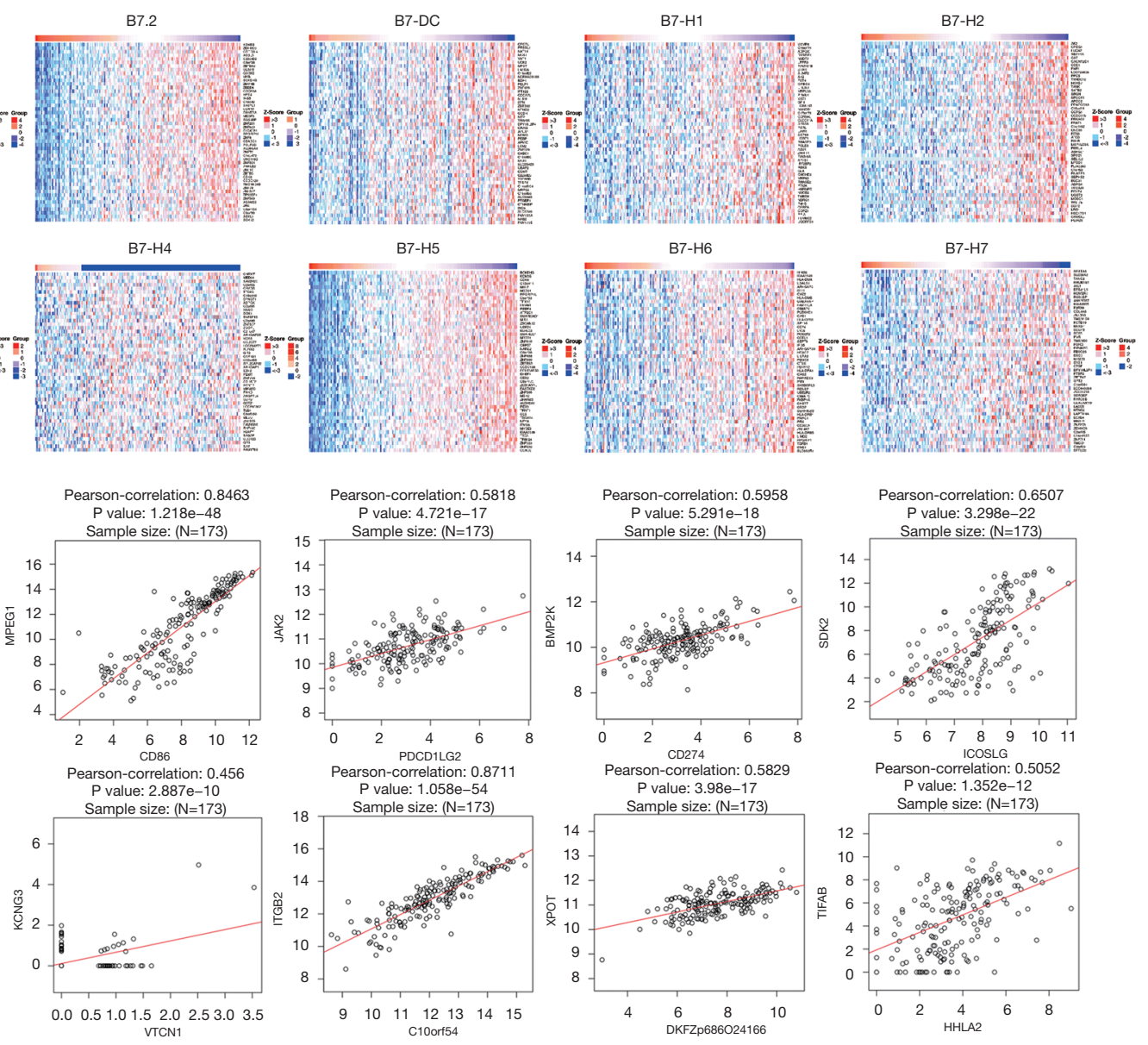

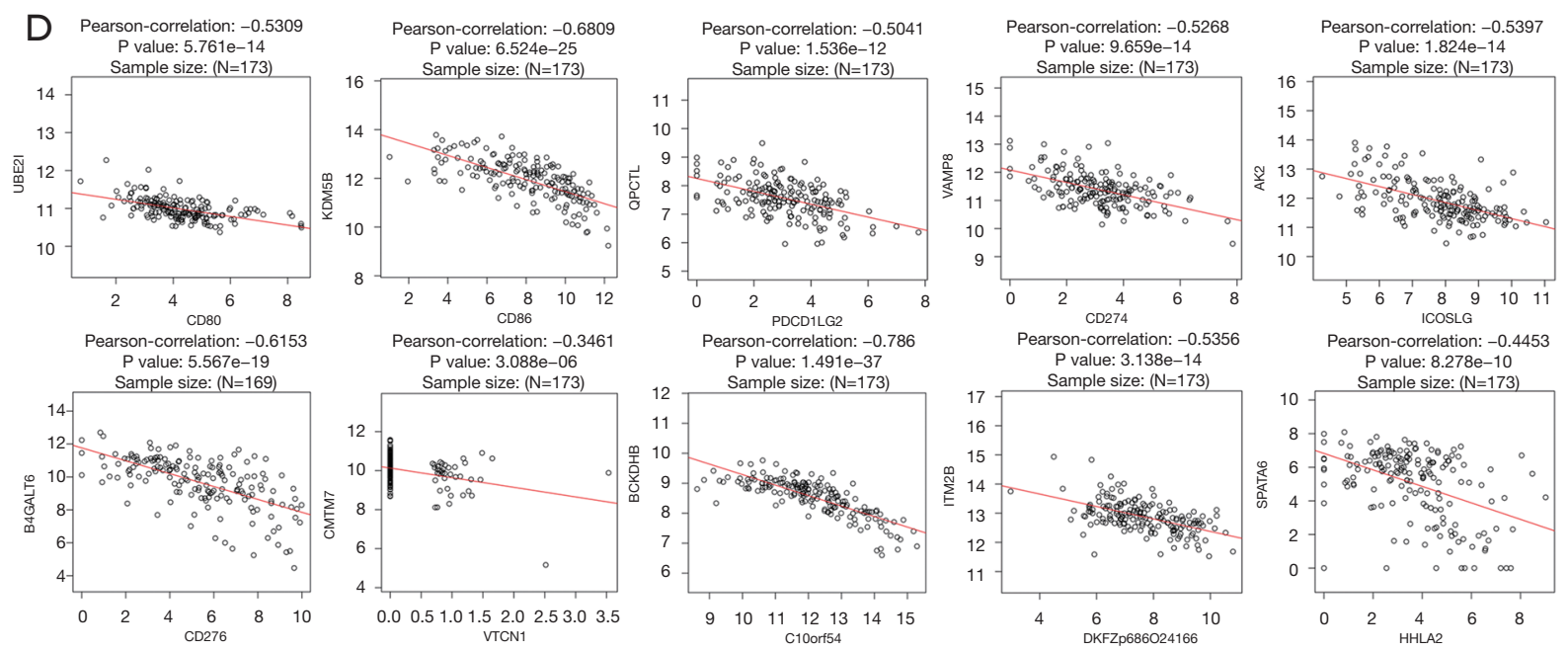

Figure 6 The significant positive and negative co-expression genes correlated with B7 family members in AML (LinkedOmics, n=173). (A,B) Heat plots showing the top 50 positive and negative co-expression genes that were correlated with B7 family members in AML, respectively. (C,D) Scatter plots showing the most positively- and negatively-correlated significant genes with B7 molecules in AML, respectively, using Pearson's correlation analysis. AML, acute myeloid leukemia.

H6, and $\mathrm{B} 7-\mathrm{H} 7$ was detected in AML cells $(11,14)$; and (IV) CD34+ AML cases exhibited a higher expression of B7-H3 (12). These suggested that a series of B7 family molecules expressed not only mRNA level but also functional protein in AML, and they might participate in AML tumor progression. In this study, the ONCOMINE database was firstly used to compare the transcriptional level of B7 molecules in cancer and normal samples. The results showed differences in the mRNA expression of different B7 family members in leukemia patients; however, most molecules did not exhibit significantly different expression between leukemia and normal samples. $B 7.1$ and $B 7-H 5$ were found to be downregulated in leukemia, while $B 7.2$ and $B$ 7- $H 2$ were over-expressed in some types of acute and chronic lymphoblastic leukemia. According to the GEPIA2 database, the mRNA expression of $B 7.2$ and $B 7-H 5$ was significantly increased, while that of $B 7.1, B 7-H 3$, and $B 7$ $H 6$ was lower in AML. These results were consistent with previous studies. Developed from FAB classification system, the accurate AML MICM classification of World Health Organization requires multidisciplinary diagnosis, including morphology, immunology, cytogenetics and molecular biology, which have the different correlation with the prognosis of AML patients (30). However, the original FAB classification remains important for identifying M3 from other subtypes, distinguishing the M5 subtype frequently with poor prognosis, etc. In this study, the expression of B7 molecules was variable for various FAB subtypes of AML, according to the UALCAN database. Interestingly, we found that several B7 molecules were significantly increased in M5, such as B7.2, B7-H3, and B7-H5. Meanwhile, in M3, a series of $\mathrm{B} 7$ members were decreased, such as $B 7.2, B 7$ $D C, B 7-H 1, B 7-H 2, B 7-H 5$, and $B 7-H 7$. Collectively, the significantly abnormal expression of $\mathrm{B} 7$ molecules might be involved in tumorigenesis in different types of AML, although this requires further investigations.

As previously reported, CD80 and CD86 expression in group I of the B7 family on AML blasts do not exhibit significant prognostic relevance (31), while ICOSL expression in AML cells influences survival by driving regulatory $\mathrm{T}$ cells expansion (11). In AML patients, an association was identified between highly expressed PDL1, PD-L2, and PD-1 in group II of the B7 family and poor OS (32). A negative prognostic correlation with B7H3 expression was reported in one study (12), while a trend towards better OS in $\mathrm{B} 7-\mathrm{H} 3$-positive patients in another study (33). These indicated that different functional protein of B7 family molecules had different clinical significance, while the prognostic value of $\mathrm{B} 7-\mathrm{H} 4, \mathrm{~B} 7-\mathrm{H} 5, \mathrm{~B} 7-\mathrm{H} 6$, and B7-H7 in AML was unclear. Some reports demonstrated that the overexpression of group III of the B7 family was associated with poor or favorable prognosis in various malignancies, including metastatic colorectal cancer (34), colorectal cancer (35), small cell lung cancer (36), and 
A

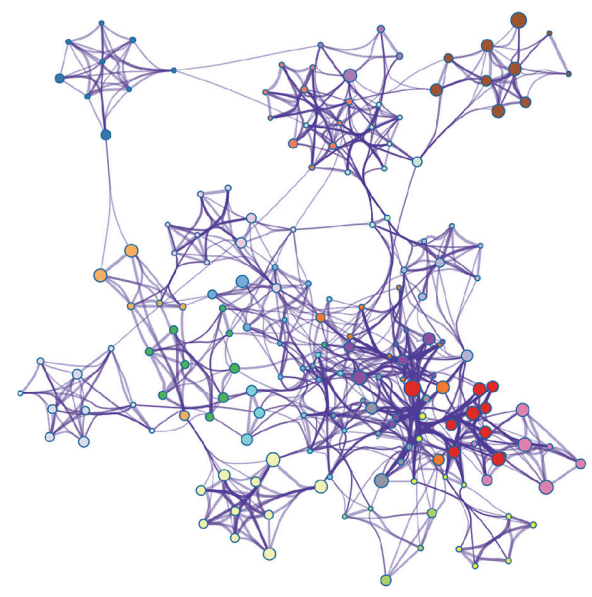

B

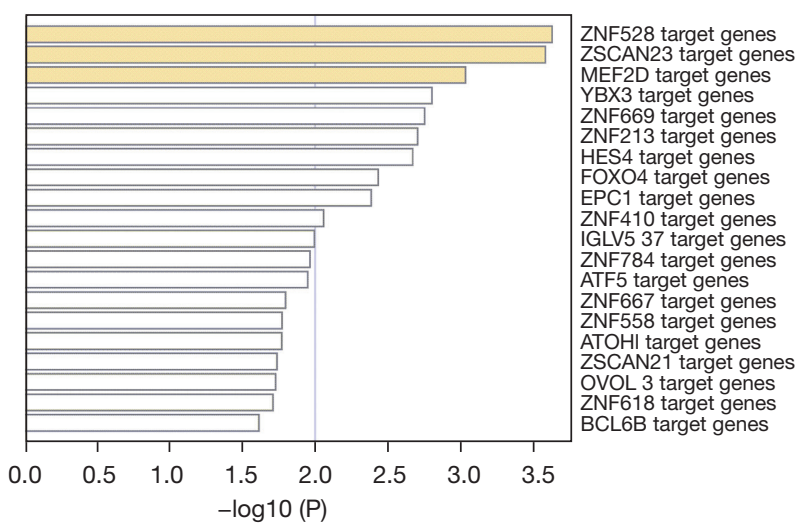

Positive regulation of cytokine production

Phagocytic vesicle

Cellular response to interferon-gamma

Response to virus

Regulation of innate immune response

Regulation of alpha-beta T cell activation

Carbohydrate derivative biosynthetic process

Immune response-regulating signaling pathway

Regulation of immune effector process

Regulation of leukocyte apoptotic process

Cell activation involved in immune response

Regulation of hemopoiesis

Cell redox homeostasis

Fatty acid transport

Lytic vacuole

Positive regulation of response to external stimulus

Cellular response to external stimulus

Small GTPase mediated signal transduction

Oxidoreductase activity

Osteoclast differentiation

\section{C}

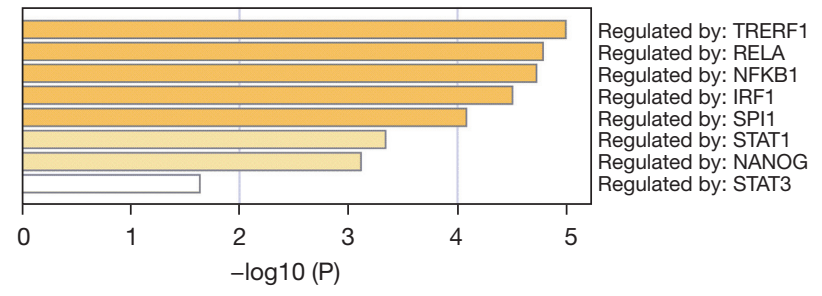

Figure 7 Enrichment analysis of B7 molecules and their significantly-associated gene alterations in AML patients (Metascape). (A) The GO and KEGG network significantly enriched terms among the B7 family and the relative gene memberships colored by functional clusters in AML. (B) Heatmap of the target transcription factors of B7 family members in AML. (C) Heatmap of the regulating genes towards the B7 family in AML. AML, acute myeloid leukemia; GO, Gene Ontology; KEGG, Kyoto Encyclopedia of Genes and Genomes.

gastric cancer (37). In mRNA level, our results confirmed the relationship between poor prognosis and aberrant high expression of $\mathrm{B} 7$ molecules in AML, including B7.1, B7$D C, B 7-H 3, B 7-H 5$, and $B 7-H 7$, which was demonstrated by one database (at least), and was analyzed by the LinkedOmics, GEPIA2, UALCAN, and TCGAportal databases. Only B 7-H6 was identified as a protective molecule for OS in AML patients. Moreover, AML patients with overexpression of $B 7-H 1$ were inclined to have a poor prognosis, although this was not statistically significant (analyzed by the LinkedOmics, GEPIA2, and TCGAportal databases, respectively). Collectively, B7 family members were associated with AML prognosis, which might be a potential therapeutic target. In addition, it was reported that many co-expression patterns of immune checkpoints were correlated with the poor OS in AML, including PD-1/ CTLA-4, PD-L2/CTLA-4, PD-1/PD-L1, PD-1/PD-L1/ PD-L2, and PD-1/LAG-3 (32). Thus, further investigations should be designed to explore the expression characteristics and clinical significance of various immune checkpoints and their combination in AML.

The present study also analyzed the genetic alteration of B7 family in AML (using the cBioPortal database). The results showed that more than half $(53.76 \%)$ of the AML cases had B7 family gene alterations, including $31.21 \%$ mRNA down-expression and $20.23 \%$ mRNA overexpression. The altered B7 group seemed to exhibit a trend toward worse OS compared with unaltered B7 group $(\mathrm{P}=0.0929)$. The relative gene alteration frequency in the altered and unaltered B7 groups was different. The most 
common gene mutations both in the altered and unaltered B7 groups were NPM1, FLT3, and DNMT3A. Previous studies have reported that many genetic mutations carried prognostic and therapeutic impact and became the main molecular markers for refining AML prognostics groups, including NPM1, FLT3, CEBPA, IDH1/2, DNMT3A, KIT, TP53, RUNX1, and ASXL1 (38,39). Particularly in the AML subgroup with concomitant NPM1 and FLT3-ITD mutations, the high PD-L1 expression could predict poor outcomes (40). We further detected the co-expression genes that were correlated with B7 family in AML (analyzed using the LinkedOmics database). The results showed that most co-expression genes were positively and negatively correlated with $\mathrm{B} 7$ molecules. $7 A K 2$ and CD274 gene amplifications were simultaneous in a case of therapyrelated myelodysplastic syndrome (MDS) evolving into AML (41). Therefore, further studies are needed to examine whether and how the genetic alteration and co-expression genes of the B7 family are correlated with tumorigenesis, progression, and prognosis in AML patients.

Moreover, we performed GO and KEGG enrichment analyses of the B7 family and 20 similar expression genes for one specific B7 molecule in AML (using the Metascape database). The results showed that a series of pathways might affect AML progression, and some pathways warrant further investigation, including the regulation of haemopoiesis, the leukocyte apoptotic process, the immune effector process, cell activation involved in the immune response, and the immune response-regulating signaling pathway. There were numerous target transcription factors of B7 family members and regulating genes towards B7 molecules. Pathogenesis or maintenance of AML might be affected by the downregulation of $Y B X 3$ induced by promoter hypermethylation, which has been demonstrated both in 94 MDS or AML cases from Australasia and in 173 AML samples from TCGA database (42). Interferons could upregulate CD40, CD80, and interferon regulatory factor 1 (IRF-1) in the M4/M5 subtypes of AML, and the RNA interference against IRF-1 could prevent interferon -gammamediated CD80 activation (43). The overexpression of SPI1 results in a reduction in the complete remission rate, relapse-free survival, and OS in cytogenetically-normal AML patients (44). This indicates that the upstream regulators and downstream effectors of B7 family members might be associated with prognosis in AML.

In conclusion, we determined that the mRNA levels of B7 family members in AML vary, and identified an association between poor prognosis in AML and the overexpression of various B7 molecules. B7 family gene alterations occurred frequently in AML cases, and the altered B7 group seemed to exhibit a trend towards worse OS. The co-expression genes and relative regulating signaling pathways of the B7 family might be involved in oncogenesis, and could be associated with prognosis in AML. Thus, the B7 family molecules could be prognostic biomarkers and therapeutic targets in AML. Further investigations need to be performed to examine the exact mechanisms through which B7 molecules contribute to tumorigenesis, and to detect the potential therapeutic effects targeting the B7 family as well as their relative signaling pathways in AML.

\section{Acknowledgments}

Funding: Natural Science Foundation of Jiangsu Province for Youths (BK20180280); Youth Innovation Foundation of The Affiliated Jiangning Hospital with Nanjing Medical University (JNYYZXKY202021); Scientific Research Foundation of The Affiliated Jiangning Hospital with Nanjing Medical University for Ph.D. (JNYYRC202101); Science and Technology Development for Social Undertakings Foundation of the Jiangning district in Nanjing (2020SHSY0101); Social Development Foundation of Zhenjiang (SH2016045).

\section{Footnote}

Reporting Checklist: The authors have completed the REMARK reporting checklist. Available at https://dx.doi. org/10.21037/atm-21-4255

Conflicts of Interest: All authors have completed the ICMJE uniform disclosure form (available at https://dx.doi. org/10.21037/atm-21-4255). The authors have no conflicts of interest to declare.

Ethical Statement: The authors are accountable for all aspects of the work in ensuring that questions related to the accuracy or integrity of any part of the work are appropriately investigated and resolved. This study was performed in accordance with the Helsinki Declaration (as revised in 2013) and was approved by the Institutional Ethics Committee of The Affiliated Jiangning Hospital with Nanjing Medical University (2020-03-034-K01). Written informed consent had already been provided by the study subjects; thus, the patient characteristics data could be retrieved from the online public databases. 
Open Access Statement: This is an Open Access article distributed in accordance with the Creative Commons Attribution-NonCommercial-NoDerivs 4.0 International License (CC BY-NC-ND 4.0), which permits the noncommercial replication and distribution of the article with the strict proviso that no changes or edits are made and the original work is properly cited (including links to both the formal publication through the relevant DOI and the license). See: https://creativecommons.org/licenses/by-nc-nd/4.0/.

\section{References}

1. Short NJ, Rytting ME, Cortes JE. Acute myeloid leukaemia. Lancet 2018;392:593-606.

2. Barrett AJ. Acute myeloid leukaemia and the immune system: implications for immunotherapy. Br J Haematol 2020;188:147-58.

3. Sanmamed MF, Chen L. A Paradigm Shift in Cancer Immunotherapy: From Enhancement to Normalization. Cell 2018;175:313-26.

4. DiNardo CD, Wei AH. How I treat acute myeloid leukemia in the era of new drugs. Blood 2020;135:85-96.

5. Seliger B, Quandt D. The expression, function, and clinical relevance of B7 family members in cancer. Cancer Immunol Immunother 2012;61:1327-41.

6. Greaves P, Gribben JG. The role of B7 family molecules in hematologic malignancy. Blood 2013;121:734-44.

7. Ni L, Dong C. New B7 Family Checkpoints in Human Cancers. Mol Cancer Ther 2017;16:1203-11.

8. Brouwer RE, Zwinderman KH, Kluin-Nelemans HC, et al. Expression and induction of costimulatory and adhesion molecules on acute myeloid leukemic cells: implications for adoptive immunotherapy. Exp Hematol 2000;28:161-8.

9. Williams P, Basu S, Garcia-Manero G, et al. The distribution of T-cell subsets and the expression of immune checkpoint receptors and ligands in patients with newly diagnosed and relapsed acute myeloid leukemia. Cancer 2019;125:1470-81.

10. Yang H, Bueso-Ramos C, DiNardo C, et al. Expression of PD-L1, PD-L2, PD-1 and CTLA4 in myelodysplastic syndromes is enhanced by treatment with hypomethylating agents. Leukemia 2014;28:1280-8.

11. Han Y, Dong Y, Yang Q, et al. Acute Myeloid Leukemia Cells Express ICOS Ligand to Promote the Expansion of Regulatory T Cells. Front Immunol 2018;9:2227.

12. $\mathrm{Hu} \mathrm{Y}, \mathrm{Lv} \mathrm{X}, \mathrm{Wu} \mathrm{Y}$, et al. Expression of costimulatory molecule $\mathrm{B} 7-\mathrm{H} 3$ and its prognostic implications in human acute leukemia. Hematology 2015;20:187-95.
13. Wang L, Jia B, Claxton DF, et al. VISTA is highly expressed on MDSCs and mediates an inhibition of T cell response in patients with AML. Oncoimmunology 2018;7:e1469594.

14. Sun X, Zhao J, Ma L, et al. B7-H6 as an efficient target for $\mathrm{T}$ cell-induced cytotoxicity in haematologic malignant cells. Invest New Drugs 2021;39:24-33.

15. Zhang W, Wang J, Wang YF, et al. Expression and Subcellular Distribution of Costimulatory Molecules B7-H1,B7-H3 and B7-H4 in Human Hematologic Malignancy Cell Lines. Zhongguo Shi Yan Xue Ye Xue Za Zhi 2016;24:1539-46.

16. Zhang W, Wang J, Wang YF, et al. B7-H3 silencing by RNAi inhibits tumor progression and enhances chemosensitivity in U937 cells. Onco Targets Ther 2015;8:1721-33.

17. Rhodes DR, Yu J, Shanker K, et al. ONCOMINE: a cancer microarray database and integrated data-mining platform. Neoplasia 2004;6:1-6.

18. Tang Z, Li C, Kang B, et al. GEPIA: a web server for cancer and normal gene expression profiling and interactive analyses. Nucleic Acids Res 2017;45:W98-102.

19. Tang Z, Kang B, Li C, et al. GEPIA2: an enhanced web server for large-scale expression profiling and interactive analysis. Nucleic Acids Res 2019;47:W556-60.

20. Chandrashekar DS, Bashel B, Balasubramanya SAH, et al. UALCAN: A Portal for Facilitating Tumor Subgroup Gene Expression and Survival Analyses. Neoplasia 2017;19:649-58.

21. Vasaikar SV, Straub P, Wang J, et al. LinkedOmics: analyzing multi-omics data within and across 32 cancer types. Nucleic Acids Res 2018;46:D956-63.

22. Gao J, Aksoy BA, Dogrusoz U, et al. Integrative analysis of complex cancer genomics and clinical profiles using the cBioPortal. Sci Signal 2013;6:pl1.

23. Zhou Y, Zhou B, Pache L, et al. Metascape provides a biologist-oriented resource for the analysis of systemslevel datasets. Nat Commun 2019;10:1523.

24. Coustan-Smith E, Song G, Clark C, et al. New markers for minimal residual disease detection in acute lymphoblastic leukemia. Blood 2011;117:6267-76.

25. Dürig J, Bug S, Klein-Hitpass L, et al. Combined single nucleotide polymorphism-based genomic mapping and global gene expression profiling identifies novel chromosomal imbalances, mechanisms and candidate genes important in the pathogenesis of T-cell prolymphocytic leukemia with inv(14)(q11q32). Leukemia 2007;21:2153-63. 
26. Choi YL, Tsukasaki K, O'Neill MC, et al. A genomic analysis of adult T-cell leukemia. Oncogene 2007;26:1245-55

27. Haferlach T, Kohlmann A, Wieczorek L, et al. Clinical utility of microarray-based gene expression profiling in the diagnosis and subclassification of leukemia: report from the International Microarray Innovations in Leukemia Study Group. J Clin Oncol 2010;28:2529-37.

28. Andersson A, Ritz C, Lindgren D, et al. Microarray-based classification of a consecutive series of 121 childhood acute leukemias: prediction of leukemic and genetic subtype as well as of minimal residual disease status. Leukemia 2007;21:1198-203.

29. Janakiram $M$, Shah UA, Liu $W$, et al. The third group of the B7-CD28 immune checkpoint family: HHLA2, TMIGD2, B7x, and B7-H3. Immunol Rev 2017;276:26-39.

30. Arber DA, Orazi A, Hasserjian R, et al. The 2016 revision to the World Health Organization classification of myeloid neoplasms and acute leukemia. Blood 2016;127:2391-405.

31. Schmohl JU, Nuebling T, Wild J, et al. Expression of 4-1BB and its ligand on blasts correlates with prognosis of patients with AML. J Investig Med 2016;64:1252-60.

32. Chen C, Liang C, Wang S, et al. Expression patterns of immune checkpoints in acute myeloid leukemia. J Hematol Oncol 2020;13:28.

33. Guery T, Roumier C, Berthon C, et al. B7-H3 protein expression in acute myeloid leukemia. Cancer Med 2015;4:1879-83.

34. Ding S, Lv X, Liu Z, et al. Overexpression of B7$\mathrm{H} 4$ is associated with infiltrating immune cells and poor prognosis in metastatic colorectal cancer. Int Immunopharmacol 2021;90:107144.

35. Zong L, Yu S, Mo S, et al. High VISTA Expression Correlates With a Favorable Prognosis in Patients With Colorectal Cancer. J Immunother 2021;44:22-8.

36. Zhang X, Xie W, Wang Z, et al. Expression of a novel

Cite this article as: Zhang W, Zhang W, Gui L, Yan X, Zhou X, Ma Y, Yang Z, Fang Y, Zhang H, Shi J. Expression and prognosis of the B7 family in acute myeloid leukemia. Ann Transl Med 2021;9(20):1530. doi: 10.21037/atm-21-4255 immune checkpoint B7-H6 ligand in human small cell lung cancer. Ann Transl Med 2020;8:589.

37. Wei L, Tang L, Chang H, et al. HHLA2 overexpression is a novel biomarker of malignant status and poor prognosis in gastric cancer. Hum Cell 2020;33:116-22.

38. Bienz M, Ludwig M, Leibundgut EO, et al. Risk assessment in patients with acute myeloid leukemia and a normal karyotype. Clin Cancer Res 2005;11:1416-24.

39. Port M, Bottcher M, Thol F, et al. Prognostic significance of FLT3 internal tandem duplication, nucleophosmin 1, and CEBPA gene mutations for acute myeloid leukemia patients with normal karyotype and younger than 60 years: a systematic review and meta-analysis. Ann Hematol 2014;93:1279-86.

40. Brodská B, Otevřelová P, Š́ak C, et al. High PD-L1 Expression Predicts for Worse Outcome of Leukemia Patients with Concomitant NPM1 and FLT3 Mutations. Int J Mol Sci 2019;20:2823.

41. Wang HY, Dell'Aquila ML, Dvanajscak Z, et al. JAK2 double minutes with resultant simultaneous amplification of JAK2 and CD274 in a therapy-related myelodysplastic syndrome evolving into an acute myeloid leukaemia. Br J Haematol 2019;185:566-70.

42. Wong JJ, Lau KA, Pinello N, et al. Epigenetic modifications of splicing factor genes in myelodysplastic syndromes and acute myeloid leukemia. Cancer Sci 2014;105:1457-63.

43. Bauvois B, Nguyen J, Tang R, et al. Types I and II interferons upregulate the costimulatory CD80 molecule in monocytes via interferon regulatory factor-1. Biochem Pharmacol 2009; 78:514-22.

44. Abo Elwafa R, Gamaleldin M, Ghallab O. The clinical and prognostic significance of FIS1, SPI1, PDCD7 and Ang2 expression levels in acute myeloid leukemia. Cancer Genet 2019;233-234:84-95.

(English Language Editor: A. Kassem) 